Research Paper

\title{
The new application of mifepristone in the relief of adenomyosis-caused dysmenorrhea
}

\author{
Xuan Che ${ }^{1,2^{*}}$, Jianzhang Wang1 ${ }^{*}$, Jiayi $\mathrm{He}^{1}$, Xinyue Guo ${ }^{1}$, Tiantian $\mathrm{Li}^{1}$, Xinmei Zhang ${ }^{1^{凶}}$ \\ 1. Women's Hospital, School of Medicine, Zhejiang University, Hangzhou, Zhejiang, P.R. China, 310006 \\ 2. Jiaxing Maternity and Child Health Care Hospital, Jiaxing, Zhejiang, P.R. China, 314000 \\ *These authors contributed equally to this work.
}

$\square$ Corresponding author: Xinmei Zhang, MD, PhD, The Department of Gynecology, Women's Hospital, School of Medicine, Zhejiang University, 1 Xueshi Road, Hangzhou, Zhejiang, P.R. China, 310006. Phone: 86-571-87061501-2131; Fax: 86-571-87061878; E-mail: zhangxinm@zju.edu.cn

(1) The author(s). This is an open access article distributed under the terms of the Creative Commons Attribution License (https://creativecommons.org/licenses/by/4.0/). See http://ivyspring.com/terms for full terms and conditions.

Received: 2019.08.12; Accepted: 2019.11.07; Published: 2020.01.14

\begin{abstract}
Background: Adenomyosis is a quite common gynecological disorder and above $30 \%$ of patients have typical secondary and progressive dysmenorrhea. Current treatments still have many disadvantages and thereby the novel treatment aiming to relieve dysmenorrhea still needs to be further investigated. Mifepristone is a wonderful drug because it is effective, safe and cheap in many diseases including adenomyosis. In this study, we aim to investigate if mifepristone could be used in the treatment of adenomyosis-associated dysmenorrhea.
\end{abstract}

Methods: Human primary endometrial epithelial and stromal cells from adenomyosis patients were isolated and treated with mifepristone. RNA-sequencing was then performed to detect the gene changes of pain-related inflammatory mediators. Meanwhile, the effect of mifepristone on the infiltration and degranulation of mast cells were investigated in adenomyosis lesions. Additionally, the role of mifepristone on the density of nerve fibers was also studied in the ectopic endometrium. At last, to evaluate the therapeutic efficacy of mifepristone on dysmenorrhea of adenomyosis, twenty participants were included and the visual analog scale (VAS) score was assessed and compared before and after treatment with mifepristone.

Results: We demonstrated that mifepristone reduced the secretion of IL-6 and TNF- $\alpha$ from endometrial epithelial and stromal cells, restricted the infiltration and degranulation of mast cells in eutopic and ectopic endometrium and decreased the density of nerve fibers by inhibiting the migration capacity of nerve cells in adenomyosis. Meanwhile, we found that mifepristone could significantly relieve dysmenorrhea of adenomyosis.

Conclusion: The findings demonstrated that mifepristone could be applied in the treatment of dysmenorrhea for the adenomyosis patients.

Key words: adenomyosis, dysmenorrhea, mifepristone, inflammation, mast cell, nerve fiber

\section{Introduction}

Adenomyosis is defined as invasion of endometrial glands and stroma into the myometrium and the prevalence of adenomyosis ranges from 8 to $27 \%$ of women in reproductive age [1]. Adenomyosis causes many health problems such as dysmenorrhea, hypermenorrhea and subfertility. Above $30 \%$ of patients have typical secondary and progressive dysmenorrhea [2]. Serious dysmenorrhea can affect the qualities of work, eating and sleep and cause depression, which restricts the daily routine of these patients and has a tremendous impact on their physical and mental health [2, 3]. Moreover, adenomyosis is diagnosed in $20-25 \%$ of infertile young women undergoing assisted reproductive technologies [4]. Severe dysmenorrhea is the primary reason for patients to choose hysterectomy and lost 
their fertility. Current therapy for adenomyosisassociated pain includes hysterectomy, oral contraceptive drugs and gonadotrophin-releasing hormone $(\mathrm{GnRH})$ agonists. However, the present medical treatment for adenomyosis-related dysmenorrhea is limited for their side effects such as premenopausal symptoms, high relapse rate after medicine withdrawal and high costs $[5,6]$. Thus, the novel medical treatment aiming to relieve dysmenorrhea should be further investigated.

The exact pathogenic mechanism of adenomyosis-related dysmenorrhea remains unclear, while inflammation and innervation possibly are the key pathogenic factors [7]. Inflammatory mediators, including IL-6, TNF- $\alpha$, IL-1 $\beta$ and IL-10, are involved in inflammatory pathway and contribute to the intense painful symptoms in adenomyosis [8-10]. Furthermore, increasing evidence supports that the activated mast cell is regarded as a director of common inflammatory pathways contributing to chronic neuropathic pain and may play a role in pathogenesis of adenomyosis [11-13]. Our previous study also showed that the recruitment and degranulation of mast cells play an important role in endometriosis-related dysmenorrhea [14]. In addition, recent research found adenomyosis-induced pain resembles neuropathic pain [15]. We also proved that the density of nerve fibers in the functional layer endometrium of adenomyosis patients was correlated with dysmenorrhea, suggesting the nerve fibers play an important role in the mechanisms of pain generation in adenomyosis [16]. Obviously, drug therapy for adenomyosis can be based on the above-mentioned pathogenesis of adenomyosis.

Mifepristone is the first and one of the most widely used selective progesterone receptor modulators (SPRM) since 1982. Besides mild adverse effect and well tolerance in its long-term clinical application, low price of this drug is another great advantage for the patients because adenomyosis needs the long-term medical therapy [17]. In China, the cost of mifepristone is only less than 4 US dollars per month while GnRH-a treatment needs more than 200 US dollars per month in the treatment of endometriosis. Currently, we find that mifepristone has more benefits for human health than what we thought before. Recent studies showed that mifepristone strongly decreased the levels of tumor necrosis factor- $\alpha$ (TNF- $\alpha)$, interleukin-1 $\beta$ (IL-1 $\beta$ ) and interleukin-6 (IL-6) of paraquat-induced lung injury in rats [18]. Treatment of mifepristone significantly downregulated the expression of neuronal nitric oxide synthase (nNOS) and N-methyl-D-aspartate receptor subunit $2 \mathrm{~B}$ (NR2B) proteins in a rat model of radicular pain [19]. Furthermore, Li et al. reported that addition of mifepristone to depot-medroxyprogesterone acetate (DMPA)-exposed endometrium significantly decreased mast tryptase-positive cells and pointed that mifepristone is associated with inhibiting the activity of mast cells [20]. Some studies in China and we also found that mifepristone could be applied in the treatment of adenomyosis. Taken together, theoretically, mifepristone may be a new therapeutic agent for adenomyosis-related pain. However, only a few studies were performed to investigate the role of mifepristone on the dysmenorrhea caused by adenomyosis.

In this study, human primary endometrial epithelial cells and stromal cells from adenomyosis patients were isolated and treated with mifepristone. RNA-sequencing was then performed to detect the gene changes of inflammatory mediators. Meanwhile, we investigated the effects of mifepristone on the infiltration and degranulation of mast cells in adenomyosis. Additionally, we investigated the role and mechanism of mifepristone on the density of nerve fibers in the ectopic endometrium of adenomyosis patients. At last, to further study the therapeutic efficacy of mifepristone on dysmenorrhea of adenomyosis, twenty participants were included and the visual analog scale (VAS) score was assessed and compared before and after treatment with mifepristone. Our study was performed to elucidate the effect of mifepristone on the relief of dysmenorrhea, which will provide a solid foundation for the application of mifepristone in the treatment of adenomyosis patients with dysmenorrhea.

\section{Materials and Methods}

\section{Isolation and identification of endometrial stromal and epithelial cells of adenomyosis}

Isolation of primary endometrial stromal and epithelial cells was performed using a previously reported method [21]. Briefly, the tissues were washed with FBS-free medium under aseptic conditions and were minced into $1 \times 1 \times 1 \mathrm{~mm}^{3}$ pieces. After the minced tissues were digested with $1 \mathrm{mg} / \mathrm{mL}$ collagenase type III at $37^{\circ} \mathrm{C}$ for $60 \mathrm{~min}$, the endometrial epithelial cells and stromal cells were separated by two sequential filtrations of 200 and 70 $\mu \mathrm{m}$ cell strainer. Endometrial epithelial cells remaining in the cell strainer were collected were cultivated in primary epithelial growth medium (PriCells, Wuhan, China) and endometrial stromal cells were cultivated in Dulbecco's modified Eagle's medium (DMEM)/F12 medium (Thermo Fisher, CA, USA) supplemented with 10\% FBS (Sigma-Aldrich, MO, USA). Identification of the isolated endometrial epithelial cells and stromal cells was assessed with 
anti-cytokeratin and anti-vimentin antibodies by immunohistochemistry as described in our previous study [22].

\section{Cell lines}

The RBL2H3 mast cell line was purchased from Stem Cell Bank, Chinese Academy of Sciences and cultivated in minimum Eagle's medium (Sigma) supplemented with $10 \%$ heat-inactivated fetal calf serum (Gibco), $100 \mathrm{U} / \mathrm{mL}$ penicillin and $100 \mu \mathrm{g} / \mathrm{mL}$ streptomycin. PC12 nerve cell line, as a neuronal model, was purchased from Chinese Academy of Sciences and cultured in a complete medium consisting of $85 \%$ F-12 medium (Sigma), 10\% heat-inactivated horse serum (Gibco), and 5\% fetal calf serum (Gibco). RBL2H3 Cells and PC12 Cells were treated with or without mifepristone at concentration of $50 \mu \mathrm{M}$ for $48 \mathrm{~h}$ in this study.

\section{The Cell-counting Kit-8 (CCK-8) Assay}

To investigate the suitable concentration of mifepristone treatment for the following study, primary endometrial epithelial cells were treated with mifepristone in different concentrations $(0,10,25,50$, 75,100 and $200 \mu \mathrm{M}$, respectively) for $24 \mathrm{~h}$. Then, the viability of above cells was detected by the CCK-8 assay (Biosharp, Beijing, China). CCK-8 reagent was added to each well and cells were incubated at $37^{\circ} \mathrm{C}$ for $2 \mathrm{~h}$ in accordance with the manufacturer's instructions. The absorbance at $450 \mathrm{~nm}$ (optical density) was detected to calculate the cell viability.

\section{High-throughput sequencing}

Based on the result of CCK-8 assay, the viability of endometrial epithelial cells was significantly decreased when the concentrations of mifepristone treatment were $75 \mu \mathrm{M}$. Subsequently, primary endometrial epithelial cells were treated with mifepristone at the concentration of $50 \mu \mathrm{M}$ for 24 hours. The epithelial cells were isolated from four independent samples. Total RNAs were isolated using Trizol reagent (Life Technologies, Grand Island, USA) and subjected to RNA high-throughput sequencing by The Beijing Genomics Institute (BGI, Shenzhen, China).

\section{Real-time polymerase chain reaction}

Total RNA of each endometrium was extracted using Trizol reagent (Takara, Japan) and reverse transcription was performed using PrimeScript Reverse Transcription reagent kit (Takara, Japan). RT-PCR was performed using SYBR Premix Ex Taq TM Kit (Takara, Japan) with ABI 7500 realtime PCR system (Thermo, MMAS, USA). The primer was designed using Primer 3 , and the nucleotide sequences of IL-6 were as follows: sense
5'-CCTCCAGAACAGATTTGAGAGTAGT-3'; and antisense 5'-GGGTCAGGGGTGGTTATTGC-3'; the nucleotide sequences of TNF-A were as follows: sense 5'-CGAGTGACAAGCCTGTAGCC-3'; and antisense 5'-TGAAGAGGACCTGGGAGTAGAT-3'. As an internal control, GAPDH was also amplified and the nucleotide sequence for the primers were as follows: sense 5'-GCCATCAATGACCCCTTCATT-3' and antisense 5'-TGACGGTGCCATGGAATTT-3.

\section{Measurement of mast cell degranulation}

As our previous study described, RBL2H3 cell degranulation was measured through the release of $\beta$-hexosaminidase [14]. RBL2H3 cells were seeded in 96-well plates $\left(5 \times 10^{4}\right.$ cells / well $)$ with or without mifepristone treatment at the concentration of $50 \mu \mathrm{M}$ for $48 \mathrm{~h}$. Then stimulated with DNP-BSA at the concentrations of $100 \mathrm{ng} / \mathrm{ml}$ (A6661, Sigma, USA) and degranulation was detected by the release of hex according to the protocol [23].

\section{Enzyme linked immunosorbent assay (ELISA)}

The endometrial epithelial cells and stromal cells were treated with mifepristone at different concentrations $(0,50$ and $100 \mu \mathrm{M}$, respectively) for 48 $h$ and the concentration of IL- 6 and TNF- $a$ protein in endometrial epithelial and stromal cells culture supernatant were detected by ELISA kits of interleukin-6 (IL-6) (ELH-IL6-1, RayBiotech, Peachtree Corners, GA, USA) and tumor necrosis factor-a (TNF-a) (ELH-TNFa-1, RayBiotech, Peachtree Corners, GA, USA). ELISA was performed according to manufacturer's instructions.

\section{Immunohistochemical staining}

Adenomyosis eutopic endometrium and corresponding ectopic endometrium were collected during surgery. The diagnosis of adenomyosis was confirmed by imaging or histological examination. Samples were collected in the proliferative phase of the menstrual cycle. Sections were incubated with anti-c-kit antibody (dilution 1:200, ab32363, Abcam, Cambridge, MA, USA) and anti-PGP9.5 antibody (dilution 1:500, Z5116, Dako Cytomation, DenmarkA/S). Immunohistochemical assay was performed as previously described [24]. Individual nerve fibers were then counted under high power $(x$ $200)$ to obtain a nerve count in a defined area. The average nerve count in five hot spots was calculated [25].

\section{Cell migration assay}

Cell migration ability was evaluated by transwell chamber assay using 24-well plates with 8.0- $\mu \mathrm{m}$ pore size membranes (BD Biosciences, CA, USA). To study the effect of mifepristone on the 
migratory ability of nerve cells, PC12 cells with or without pre-treatment of mifepristone at the concentration $50 \mu \mathrm{M}$ were added into the upper chamber of the insert in $200 \mu \mathrm{L}$ of serum-free medium, while the lower chamber contained growth media with $10 \%$ FBS. After $24 \mathrm{~h}$ incubation, cells in the upper chamber were removed with a cotton swab and the migrated cells in the lower chamber were fixed with methanol, stained with crystal violet and counted with a microscope (Olympus, Japan). The PC12 cells that passed through the membrane was defined as migrated cells.

\section{Patients and clinical evaluation}

This study was approved by Ethics Committee of Women's Hospital, School of Medicine, Zhejiang University and registered in Chinese Clinical Trial Registry (1800015514). Twenty cases of adenomyosis patients were included after informed consent. No hormone or similar drugs were used for 6 months before treatment. The patients were treated with mifepristone by oral administration at $5 \mathrm{mg}$ per day. The visual analog scale (VAS) was used to evaluate the degree of dysmenorrhea before and after treatment of mifepristone for 3 months. The left end of the VAS was scored as 0 to represent "no pain" while the right end was scored as 10, representing the "most severe pain imagined" [26]. The VAS score was self-assessed by each patient prior to treatment. On the other hand, platelet count in serum of adenomyosis patients was obtained, and they were analyzed in the hematology laboratory of our hospital.

\section{Statistical Analysis}

SPSS program version 19.0 and Graph Pad Prism 5 software were applied for statistical analysis. Data are shown as the mean \pm Standard Error of Mean (SEM). P values were determined by the two-tailed Student's $t$ test or Mann-Whitney $U$ test when comparing two groups and by a one-way ANOVA when comparing more than two groups. Statistical difference was considered to be significant at a value of $\mathrm{P}<0.05\left(^{*}\right)$, highly significant at a value of $\mathrm{P}<0.01$ $\left.{ }^{* *}\right)$ and extremely significant when $\left.\mathrm{P}<0.001{ }^{* * *}\right)$.

\section{Results}

\section{Mifepristone reduces the secretion of IL-6 and TNF- $\alpha$ from endometrial epithelial and stromal cells in adenomyosis.}

To investigate the potential mechanism of mifepristone relieving dysmenorrhea on the adenomyosis, RNA-sequencing was performed to detect the changes of gene expression in the primary endometrial epithelial cells with or without treatment of mifepristone. Firstly, CCK-8 assay was performed to determine the effective concentrations of mifepristone on the primary endometrial epithelial cells of adenomyosis. Cells were treated with mifepristone at different concentrations $(0,10,25,50$, 75,100 and $200 \mu \mathrm{M}$, respectively) for $24 \mathrm{~h}$. As shown in Fig. 1A, the viability of endometrial epithelial cells was significantly decreased when treated with mifepristone at concentrations above $50 \mu \mathrm{M}$. The effective concentration of mifepristone applied in this study was similar to that used in treatments of kinds of cancers [27]. Based on the result of CCK-8 assay, the endometrial epithelial cells were treated with mifepristone at the concentration of $50 \mu \mathrm{M}$ for 24 hours $(n=4)$ and gene expression was examined by RNA-sequencing. Fig.1B showed that mifepristone significantly down-regulated the expressions of IL-6 and TNF-A in endometrial epithelial cells when compared to controls, which are the important pro-inflammatory chemokines closely correlated with dysmenorrhea.

Then, the down-regulations of IL-6 and TNF-a in the mifepristone-treated group were further confirmed by qRT-PCR and ELISA not only in primary endometrial epithelial cells but also in stromal cells. The mRNA expression of IL-6 and TNF-A was decreased in both endometrial epithelial cells and stromal cells when treated with mifepristone treatment in a dose-dependent manner. Subsequently, ELISA assay was conducted to detect the concentrations of IL-6 and TNF- $\mathrm{a}$ in cell culture supernatants of endometrial epithelial cells and stromal cells with and without mifepristone treatment. Endometrial epithelial cells and stromal cells were treated with mifepristone at different concentrations $(0,50$ and $100 \mu \mathrm{M})$ for $48 \mathrm{~h}$. We found that the concentrations of IL-6 and TNF-a in cell culture supernatants were significantly decreased in both endometrial epithelial and stromal cells when treated with mifepristone in a dose-dependent manner (Fig. 1C). These results suggested that mifepristone reduces the secretion of IL- 6 and TNF- $\alpha$ from endometrial epithelial and stromal cells in adenomyosis and therefore may have an effect on the relief of pain for the adenomyosis patients.

\section{Mifepristone inhibits the infiltration and activity of degranulation of mast cells in adenomyosis}

As well known, mast cells mediate neurogenic inflammation and pain [28]. The activated and degranulating mast cells may exert indirect effects on the development of neuropathic pain [29]. 
A

Endometrial Epithelial Cells

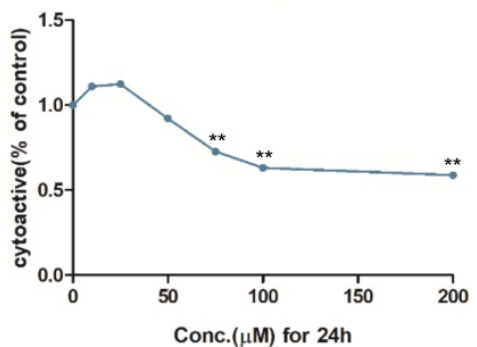

B

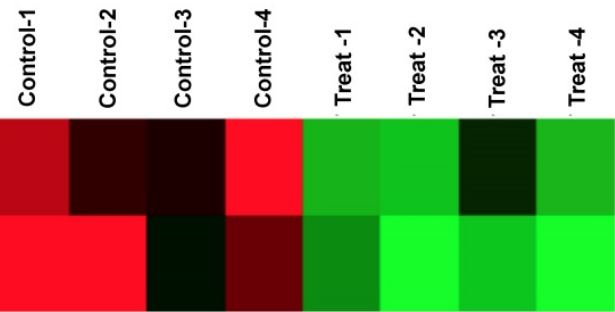

IL-6

TNF-a

C

Endometrial Epithelial Cells

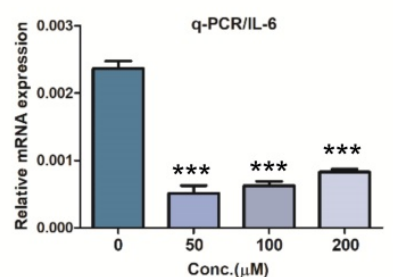

Endometrial Stromal Cells

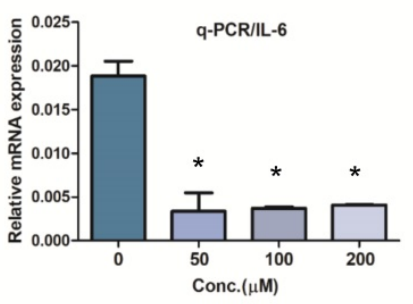

Endometrial Epithelial Cells

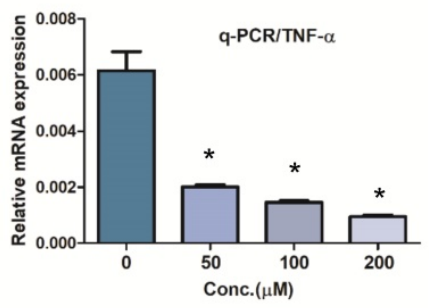

Endometrial Stromal Cells

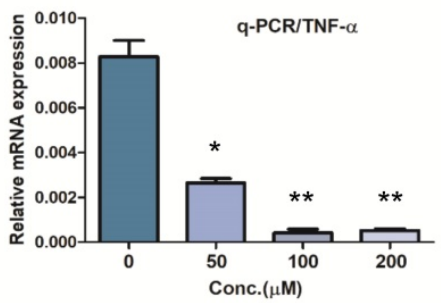

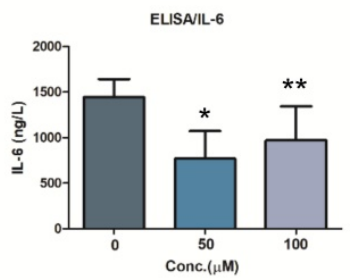
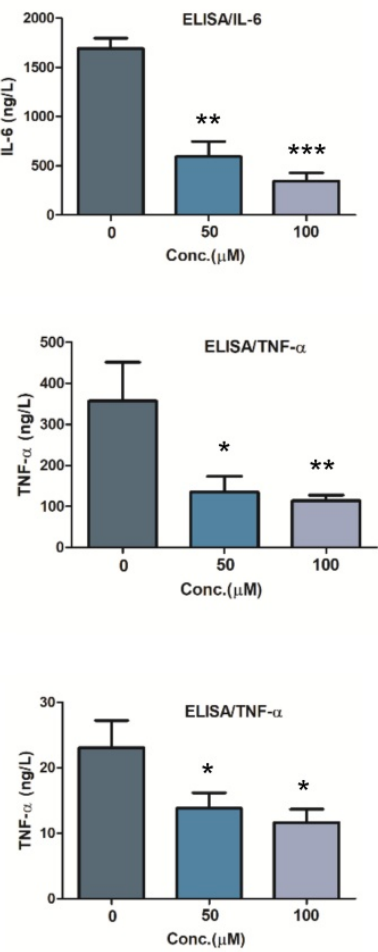

Figure 1. Mifepristone reduces the secretion of IL-6 and TNF- $\alpha$ from endometrial epithelial and stromal cells in adenomyosis. (A) Human primary endometrial epithelial cells were treated with mifepristone in different concentrations for $24 \mathrm{~h}$, and CCK-8 assay was performed. The viability of endometrial epithelial cells was significantly decreased when treated with mifepristone at $75 \mu \mathrm{M}$ while there was no significant difference at $50 \mu \mathrm{M}$. Concentration at $50 \mu \mathrm{M}$ was therefore selected for the following RNA-sequencing. (B) Primary endometrial epithelial cells were treated with mifepristone at the concentration of $50 \mu M$ and then subjected to next generation sequencing. The endometrial epithelial cells were from four biologically independent samples and the data were shown in quadruplicate. (C) qRT-PCR and ELISA were performed to detect the role of mifepristone on the down-regulations of IL-6 and TNF- $\alpha$ in endometrial epithelial and stromal cells in different concentrations. Data were shown as mean \pm SEM. $* \mathrm{P}<0.05, * * \mathrm{P}<0.01$ and $* * * \mathrm{P}<0.001$. 
A
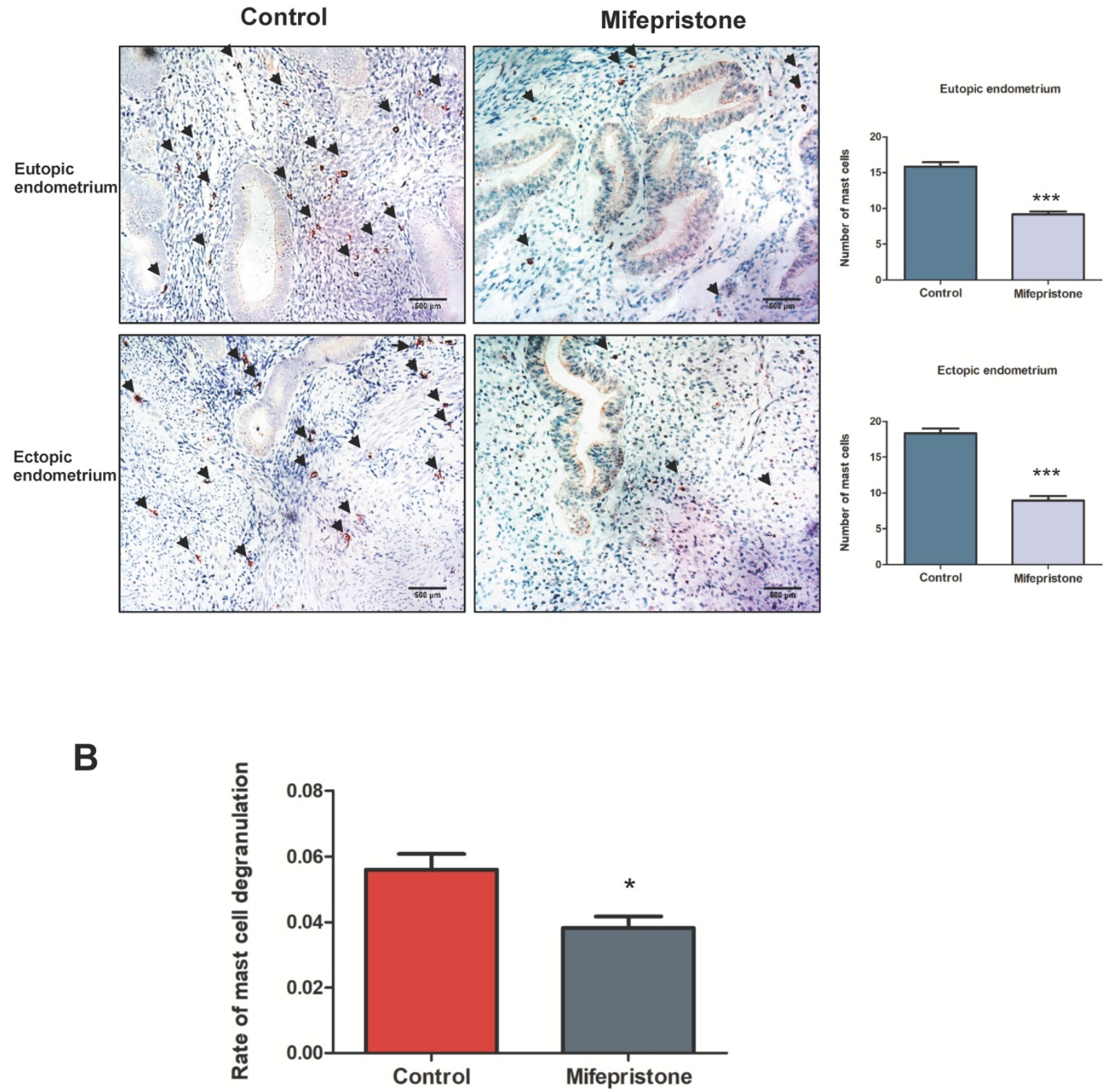

Figure 2. Mifepristone decreased the number of mast cells in both eutopic and ectopic endometriums and also inhibited the activity of degranulation. (A) Immunohistochemical staining for c-kit was examined in the eutopic and ectopic endometriums with or without mifepristone treatment. The black arrow indicates the mast cells. Scale bars $=500 \mu \mathrm{m}$. Image was captured at $200 \times$ magnification. (B) Rate of active degranulation in RBL2H3 mast cells was examined after treatment of $50 \mu \mathrm{M}$ of mifepristone for 48h. Data were expressed as mean \pm SEM. Statistical difference was determined by Mann-Whitney $U$ test. $* \mathrm{P}<0.05, * * \mathrm{P}<0.01$ and $* * * \mathrm{P}<0.001$

To study the effect of mifepristone on the mast cell-infiltration in adenomyosis, immunohistochemistry was conducted to detect the number of mast cells by staining with c-kit in eutopic and ectopic endometriums of adenomyosis. We observed the numbers of mast cells were significantly decreased in both eutopic and ectopic endometriums after mifepristone treatment $(\mathrm{P}<0.001$; Fig. 2A). To further determine whether mifepristone has effect on the activity of degranulation of mast cells. RBL2H3 mast cells were treated with mifepristone at concentration of $50 \mu \mathrm{M}$ for $48 \mathrm{~h}$. The rate of degranulation of RBL2H3 cells treated with mifepristone was significantly decreased when compared to mifepristone-untreated group ( $p<0.05$; Fig. $2 B$ ). The above results revealed that mifepristone inhibits the infiltration and the activity of degranulation of mast cells in both eutopic and ectopic endometriums of adenomyosis.

\section{Mifepristone decreases the density of nerve fibers in both eutopic and ectopic endometriums of adenomyosis}

It is known that afferent sensory fibers are critical for the conduction of adenomyosis-caused 
pain. Our previous study also found that dysmenorrhea was positively correlated with the density of PGP 9.5-immunoactive nerve fibers in the basal layer of the endometrium and myometrium [30]. To investigate the effect of mifepristone on the innervation in adenomyosis, immunohistochemistry was conducted to detect PGP 9.5-immunoactive nerve fibers in endometrium and myometrium tissue with and without mifepristone treatment. Fig. 3A showed that the density of PGP 9.5-immunoactive nerve fibers in mifepristone-treated adenomyosis group was significantly decreased in both eutopic and ectopic endometrium when compared to mifepristone-untreated group, especially in ectopic endometrium of adenomyosis. The findings suggested that mifepristone reduces the density of nerve fibers in adenomyosis, which may play an important role in relieving adenomyosis-caused pain.
A
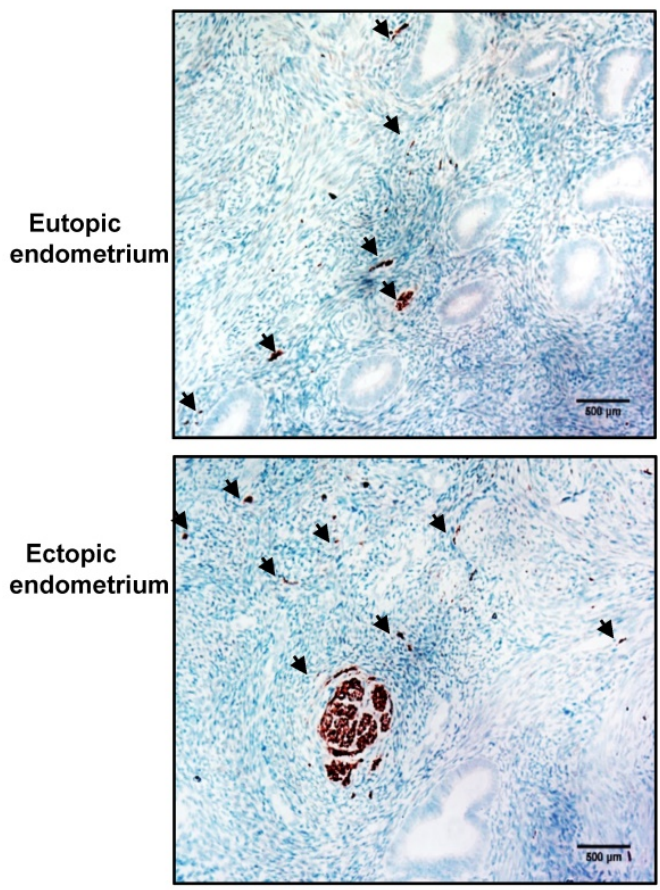
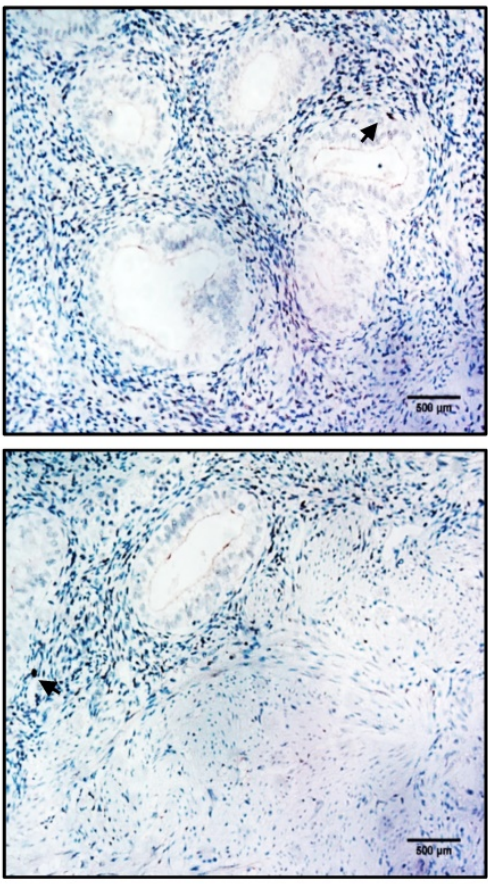

Eutopic endometrium/PGP 9.5

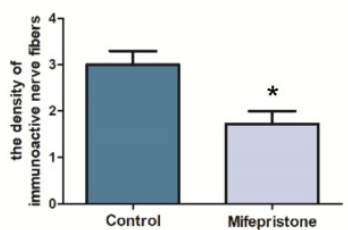

Ectopic endometrium/PGP 9.5

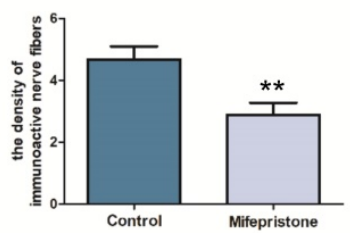

B

Control

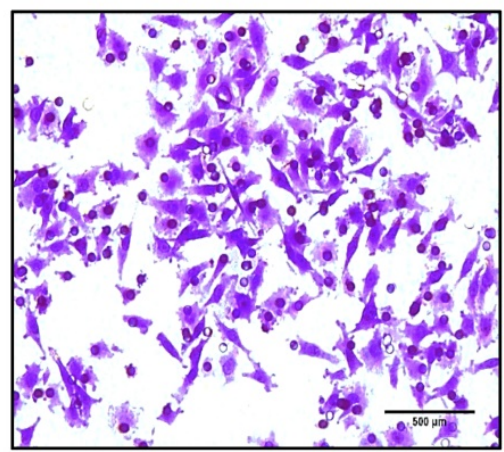

Mifepristone

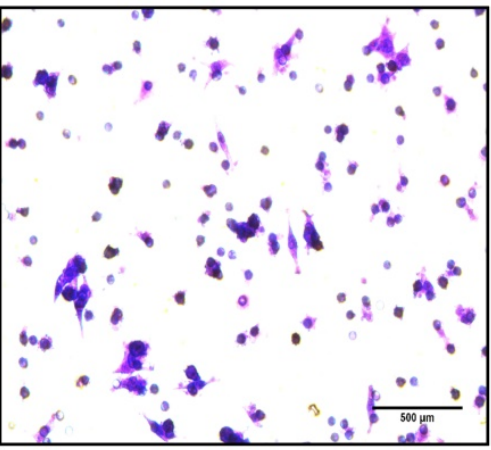

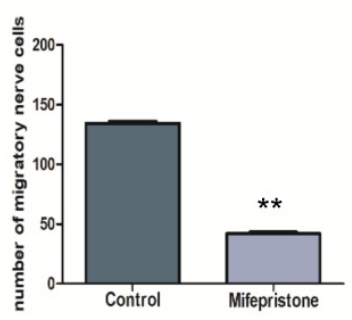

Figure 3. Mifepristone decreases the density of nerve fibers by inhibiting the migratory capacity of nerve cells in adenomyosis. (A) Nerve fibers were stained by immunohistochemical staining using PGP9.5 antibody in both eutopic and ectopic endometriums with or without mifepristone treatment. Scale bars $=500 \mu M$. Image was captured at 200x magnification. (B) Phase-contract images of migrated PC12 nerve cells on the bottom of transwell insert membrane with or without treatment of mifepristone. Number of migrated $\mathrm{PC} 12$ nerve cells on the bottom of PET membrane was counted as indicated conditions. Data were expressed as mean $\pm \mathrm{SEM}$. $* \mathrm{P}<0.05$, $* * \mathrm{P}<0.01$ and $* * * \mathrm{P}<0.001$. 
A

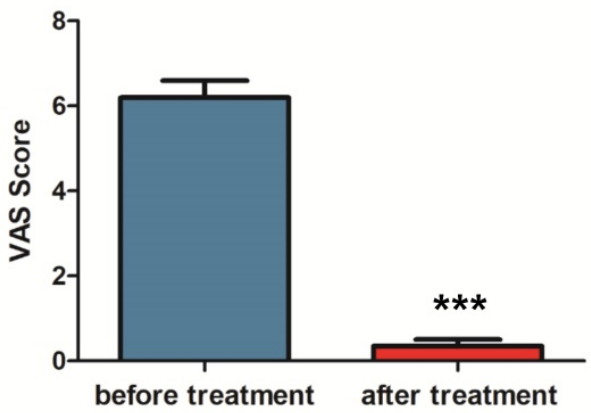

B

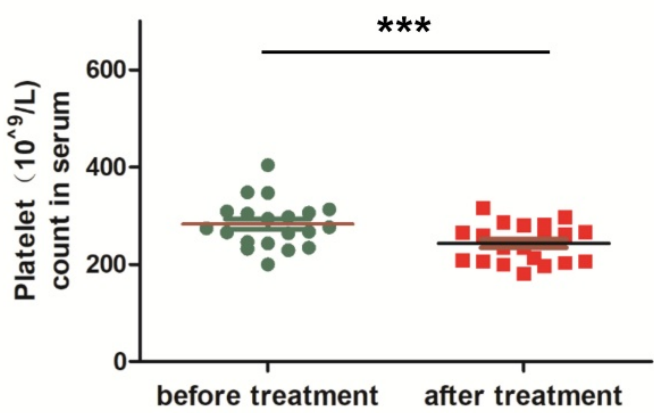

Figure 4. Mifepristone significantly relieved dysmenorrhea in adenomyosis patients. (A) The visual analog scale (VAS) score was applied for the pain assessment of dysmenorrhea in the included patients. The VAS score was significantly decreased after mifepristone treatment for the adenomyosis patients. (B) The platelet count in serum of adenomyosis patient was measured before and after three-month treatment with mifepristone. Data were expressed as mean $\pm \mathrm{SEM}$. $* \mathrm{P}<0.05$, $* * \mathrm{P}<0.01$ and $* * * \mathrm{P}<0.001$.

\section{Mifepristone decreases the density of nerve fibers by inhibiting the migration capacity of nerve cells in adenomyosis}

To investigate the potential mechanism of how mifepristone decreased the density of nerve fibers in adenomyosis, migration assay was performed to detect the effect of mifepristone on the migratory capacity of PC12 nerve cells in adenomyosis. As shown in Fig. 3B, the number of migratory PC12 cells was significantly decreased in mifepristone-treated group when compared to untreated group in a dose-dependent manner $(\mathrm{p}<0.000)$, demonstrating that the migratory ability of nerve cells was significantly restricted after treatment with mifepristone. The above data indicated mifepristone decreases the density of nerve fibers by inhibiting the migratory capacity of nerve cells in adenomyosis.

\section{Mifepristone significantly relieves dysmenorrhea in adenomyosis patients}

To study the therapeutic efficacy of mifepristone on dysmenorrhea of adenomyosis patients, the VAS score was applied for pain assessment of dysmenorrhea. The VAS score was assessed and compared for the same patient before and after treatment with mifepristone. As shown in Fig. 4A, mifepristone treatment significantly decreased the VAS score of dysmenorrhea for adenomyosis patients when compared to pre-treatment. In addition, it is known that the platelet count in serum is an important marker for the development of adenomyosis and closely associated with dysmenorrhea symptoms. The platelet count in serum before treatment was $282.66 \pm 10.8410^{\wedge} 9 / \mathrm{L}$ while the mean concentration was $242.95 \pm 8.8010^{\wedge 9} / \mathrm{L}$ (Fig.4B), indicating that the platelet count in serum were significantly reduced after mifepristone treatment. Therefore, we concluded that mifepristone could relieve dysmenorrhea symptoms for adenomyosis patients.

\section{Discussion}

Dysmenorrhea is a common symptom in adenomyosis and is one of the main reasons that women seek medical treatment. Although medical therapies such as GnRH-a, medroxyprogesterone acetate (MPA) and danazol have shown certain clinical effects for relieving adenomyosis-related dysmenorrhea, the potential side effects compromise those clinical applications. Afferent sensory fibers and proinflammatory mediators are correlated with adenomyosis pain, which can be considered an inflammatory neuropathic pain. Recent studies showed that mifepristone may play important roles in the development of neuropathic pain diseases. However, the evidence for guiding clinical use of mifepristone treatment is insufficient in adenomyosis. The present study will elucidate the feasibility of this old drug for new use in adenomyosis.

Inflammation is a major biological determinant in the pathogenesis of adenomyosis and proinflammatory/inflammatory cytokines act as chemical neurotransmitters to stimulate uterine contraction and cause dysmenorrhea [31]. In the present study, we found that mifepristone reduces the secretion of IL-6 and TNF- $\alpha$ from endometrial epithelial and stromal cells in adenomyosis. Similar to MPA and danazol treatment of adenomyosis [32, 33], mifepristone treatment inhibited the secretion of IL- 6 in endometrial epithelial and stromal cells of adenomyosis in our experiments. Yang et al. reported that MPA and danazol have no effect on the suppression of TNF-a by endometrial and stromal cells in adenomyosis [34] while our data showed mifepristone significantly decreased the mRNA and protein expression of TNF- $\alpha$ in both endometrial 
epithelial and stromal cells of adenomyosis. Recent reports pointed that selective progesterone receptor modulators may be possibly more effectively than progestins in relieving adenomyosis-associated pain, but the underlying mechanism was still unclear [35, 36]. Our findings showed that mifepristone significantly decreased the expressions of IL- 6 and TNF-a in both endometrial epithelial and stromal cells of adenomyosis, which may be the reason that mifepristone is more effectively than progestins in the relief of adenomyosis-associated pain.

Increasing evidence supports that activated and degranulating mast cells play an important role in the development of pain, hyperalgesia and dysmenorrhea $[37,38]$. Our previous study also demonstrate that the activity and degranulation of mast cells play an important role in endometriosis-related dysmenorrhea [14]. Moreover, it is reported that mast cells contribute to the development of inflammation in adenomyosis [10]. Therefore, those drugs that can inhibit mast cell-activation and suppress mast cell-degranulation may be used as effective therapeutic agents for adenomyosis. As we expected, our study showed that the infiltration of mast cells was significantly decreased in both eutopic and ectopic endometriums after mifepristone treatment. Moreover, the rate of degranulation of mast cells treated with mifepristone were decreased when compared to mifepristone-untreated group. Hence, we concluded that mifepristone relieved the dysmenorrhea symptom of adenomyosis patients through inhibiting the infiltration and the activity of degranulation of mast cells in eutopic and ectopic endometriums.

It is well known that pain is mediated by sensory nerves. Afferent sensory fibers and proinflammatory mediators are correlated with adenomyosis pain. Our previous study found that the distribution of nerve fibers in the ectopic endometrium play an important role on the pain symptoms in both endometriosis and adenomyosis [16]. The present study found that mifepristone decreases the density of nerve fibers in both eutopic and ectopic endometriums of adenomyosis. Furthermore, Transwell assay was then performed to confirm that mifepristone decreased the migration of nerve cells in a dose-dependent manner. Taken together, our data suggested that the relief of adenomyosis-associated dysmenorrhea by mifepristone is related to the decrease of density of nerve fibers by inhibiting the migration capacity of nerve cells in adenomyosis.

At last, the efficacy of mifepristone treatment in adenomyosis was further confirmed by comparing the pain assessment of dysmenorrhea in the same adenomyosis patient before and after mifepristone treatment. We concluded that mifepristone effectively relieved dysmenorrhea symptoms for adenomyosis patients. Furthermore, it is reported that platelets played an important role in the development of adenomyosis and anti-platelet treatment could reduce uterine hyperactivity and improve generalized hyperalgesia [39]. Our data showed that mifepristone significantly decreased platelet count in serum of the adenomyosis patients. Therefore, the clinical results further proved that mifepristone was efficient in the treatment of adenomyosis-associated dysmenorrhea and the effect of treatment in adenomyosis is similar to endometriosis $[40,41]$.

\section{Conclusion}

We firstly demonstrated that mifepristone reduced the secretion of IL-6 and TNF- $\mathrm{a}$ from endometrial epithelial and stromal cells, restricted the infiltration and degranulation of mast cells in eutopic and ectopic endometrium and decreased the density of nerve fibers by inhibiting the migratory capacity of nerve cells in adenomyosis. Meanwhile, we found that mifepristone could significantly relieve adenomyosis-associated dysmenorrhea. The findings demonstrated that mifepristone could be applied in the treatment of dysmenorrhea for the adenomyosis patients.

\section{Acknowledgments}

We deeply appreciate that the study was funded by National Key R\&D Program of China (Grant number: 2017YFC1001202) and National Natural Science Foundation of China (Grant numbers: 81671429 and 81802591).

\section{Competing Interests}

The authors have declared that no competing interest exists.

\section{References}

1. Naftalin J, Hoo W, Pateman K, Mavrelos D, Holland T, Jurkovic D. How common is adenomyosis? A prospective study of prevalence using transvaginal ultrasound in a gynaecology clinic. Hum Reprod. 2012; 27: 3432-9.

2. Garcia L, Isaacson K. Adenomyosis: review of the literature. J Minim Invasive Gynecol. 2011; 18: 428-37.

3. Pinzauti S, Lazzeri L, Tosti C, Centini G, Orlandini C, Luisi S, et al. Transvaginal sonographic features of diffuse adenomyosis in 18-30-year-old nulligravid women without endometriosis: association with symptoms. Ultrasound Obstet Gynecol. 2015; 46: 730-6.

4. Puente JM, Fabris A, Patel J, Patel A, Cerrillo M, Requena A, et al. Adenomyosis in infertile women: prevalence and the role of $3 \mathrm{D}$ ultrasound as a marker of severity of the disease. Reprod Biol Endocrinol. 2016; 14: 60.

5. Yang X, Zhang X, Lin B, Feng X, Aili A. Combined therapeutic effects of HIFU, GnRH-a and LNG-IUS for the treatment of severe adenomyosis. Int J Hyperthermia. 2019; 36: 486-92.

6. Radzinsky VE, Khamoshina MB, Nosenko EN, Dukhin AO, Sojunov MA, Orazmuradov AA, et al. Treatment strategies for pelvic pain associated with adenomyosis. Gynecol Endocrinol. 2016; 32: 19-22.

7. Anaf V, Simon P, El Nakadi I, Fayt I, Simonart T, Buxant F, et al. Hyperalgesia, nerve infiltration and nerve growth factor expression in deep adenomyotic nodules, peritoneal and ovarian endometriosis. Hum Reprod. 2002; 17: 1895-900. 
8. Qin X, Zhang H, Wang F, Xue J, Wen Z. Expression and possible role of interleukin-10 receptors in patients with adenomyosis. Eur J Obstet Gynecol Reprod Biol. 2012; 161: 194-8.

9. Li B, Chen M, Liu X, Guo SW. Constitutive and tumor necrosis factor-alpha-induced activation of nuclear factor-kappaB in adenomyosis and its inhibition by andrographolide. Fertil Steril. 2013; 100: 568-77.

10. Carrarelli P, Yen CF, Funghi L, Arcuri F, Tosti C, Bifulco G, et al. Expression of Inflammatory and Neurogenic Mediators in Adenomyosis. Reprod Sci. 2017; 24: 369-75.

11. Graziottin A, Skaper SD, Fusco M. Mast cells in chronic inflammation, pelvic pain and depression in women. Gynecol Endocrinol. 2014; 30: 472-7.

12. Sa RN, Song JH. Relationship between ultrastructural features with the expression of connexin 43 in the uterine junction zone and pathogenesis of adenomyosis. Zhonghua Fu Chan Ke Za Zhi. 2010; 45: 762-6.

13. Dong H, Zhang X, Qian Y. Mast cells and neuroinflammation. Med Sci Monit Basic Res. 2014; 20: 200-6.

14. Zhu TH, Ding SJ, Li TT, Zhu LB, Huang XF, Zhang XM. Estrogen is an important mediator of mast cell activation in ovarian endometriomas. Reproduction. 2018; 155: 73-83.

15. Chen Y, Zhu B, Zhang H, Ding D, Liu X, Guo SW. Possible Loss of GABAergic Inhibition in Mice With Induced Adenomyosis and Treatment With Epigallocatechin-3-Gallate Attenuates the Loss With Improved Hyperalgesia. Reprod Sci. 2014; 21: 869-82.

16. Zhang $\mathrm{X}, \mathrm{Lu} \mathrm{B}$, Huang $\mathrm{X}, \mathrm{Xu} \mathrm{H}$, Zhou $\mathrm{C}$, Lin J. Endometrial nerve fibers in women with endometriosis, adenomyosis, and uterine fibroids. Fertil Steril. 2009; 92: 1799-801

17. Spitz IM, Grunberg SM, Chabbert-Buffet N, Lindenberg T, Gelber H, Sitruk-Ware R. Management of patients receiving long-term treatment with mifepristone. Fertil Steril. 2005; 84: 1719-26.

18. Hong GL, Cai QQ, Tan JP, Jiang XZ, Zhao GJ, Wu B, et al. Mifepristone-inducible recombinant adenovirus attenuates paraquat-induced lung injury in rats. Hum Exp Toxicol. 2015; 34: 32-43.

19. Zhang J, Zhang W, Sun Y, Liu Y, Song L, Ma Z, et al. Activation of GRs-Akt-nNOs-NR2B signaling pathway by second dose GR agonist contributes to exacerbated hyperalgesia in a rat model of radicular pain. Mol Biol Rep. 2014; 41: 4053-61.

20. Li A, Felix JC, Yang W, Xiong DW, Minoo P, Jain JK. Effect of mifepristone on endometrial matrix metalloproteinase expression and leukocyte abundance in new medroxyprogesterone acetate users. Contraception. 2007; 76: 57-65.

21. Chan RW, Schwab KE, Gargett CE. Clonogenicity of human endometrial epithelial and stromal cells. Biol Reprod. 2004; 70: 1738-50.

22. Ding S, Zhu T, Tian Y, Xu P, Chen Z, Huang X, et al. Role of Brain-Derived Neurotrophic Factor in Endometriosis Pain. Reprod Sci. 2018; 25: 1045-57.

23. Kuehn HS, Radinger M, Gilfillan AM. Measuring mast cell mediator release. Curr Protoc Immunol. 2010; Chapter 7: Unit7 38.

24. Hu Y, Wu AY, Xu C, Song KQ, Wang WJ, Yin X, et al. MicroRNA-449a Inhibits Tumor Metastasis through AKT/ERK1/2 Inactivation by Targeting Steroid Receptor Coactivator (SRC) in Endometrial Cancer. J Cancer. 2019; 10: 547-55.

25. Barcena de Arellano ML, Oldeweme J, Arnold J, Schneider A, Mechsner S. Remodeling of estrogen-dependent sympathetic nerve fibers seems to be disturbed in adenomyosis. Fertil Steril. 2013; 100: 801-9.

26. Li L, Leng J, Jia S, Lang J. Treatment of symptomatic adenomyosis with the levonorgestrel-releasing intrauterine system. Int J Gynaecol Obstet. 2019.

27. Li A, Felix JC, Minoo P, Amezcua CA, Jain JK. Effect of mifepristone on proliferation and apoptosis of Ishikawa endometrial adenocarcinoma cells. Fertil Steril. 2005; 84: 202-11.

28. Green DP, Limjunyawong N, Gour N, Pundir P, Dong X. A Mast-Cell-Specific Receptor Mediates Neurogenic Inflammation and Pain. Neuron. 2019; 101: 412-20 e3.

29. Biedermann T, Kneilling M, Mailhammer R, Maier K, Sander CA, Kollias G, et al. Mast cells control neutrophil recruitment during $\mathrm{T}$ cell-mediated delayed-type hypersensitivity reactions through tumor necrosis factor and macrophage inflammatory protein 2. J Exp Med. 2000; 192: 1441-52.

30. Zhang $X$, Lu B, Huang $X, X u H$, Zhou C, Lin J. Innervation of endometrium and myometrium in women with painful adenomyosis and uterine fibroids. Fertil Steril. 2010; 94: 730-7.

31. Jiang C, Cheng Z. Update of recent studies of adenomyosis-associated dysmenorrhea. Gynecology and Minimally Invasive Therapy. 2016; 5: 137-40.

32. Hong SC, Khoo CK. An update on adenomyosis uteri. Gynecology and Minimally Invasive Therapy. 2016; 5: 106-8.

33. Vannuccini S, Luisi S, Tosti C, Sorbi F, Petraglia F. Role of medical therapy in the management of uterine adenomyosis. Fertil Steril. 2018; 109:398-405.

34. Yang JH, Chen MJ, Wu MY, Chen YC, Yang YS, Ho HN. Decreased suppression of interleukin-6 after treatment with medroxyprogesterone acetate and danazol in endometrial stromal cells of women with adenomyosis. Fertil Steril. 2006; 86: 1459-65.

35. Bouchard P, Chabbert-Buffet N, Fauser BC. Selective progesterone receptor modulators in reproductive medicine: pharmacology, clinical efficacy and safety. Fertil Steril. 2011; 96: 1175-89.

36. Tsui KH, Lee WL, Chen CY, Sheu BC, Yen MS, Chang TC, et al. Medical treatment for adenomyosis and/or adenomyoma. Taiwan J Obstet Gynecol. 2014; 53: 459-65.

37. D'Cruz OJ, Uckun FM. Targeting mast cells in endometriosis with janus kinase 3 inhibitor, JANEX-1. Am J Reprod Immunol. 2007; 58: 75-97.
38. Tsuji $\mathrm{S}$, Tsuji $\mathrm{K}$, Otsuka $\mathrm{H}$, Murakami T. Increased mast cells in endocervical smears of women with dysmenorrhea. Cytojournal. 2018; 15: 27.

39. Zhu B, Chen Y, Shen X, Liu X, Guo SW. Anti-platelet therapy holds promises in treating adenomyosis: experimental evidence. Reprod Biol Endocrinol. 2016; 14: 66 .

40. Zhang YX. Effect of mifepristone in the different treatments of endometriosis. Clin Exp Obstet Gynecol. 2016; 43: 350-3.

41. Xue HL, Yu N, Wang J, Hao WJ, Li Y, Liu MY. Therapeutic effects of mifepristone combined with Gestrinone on patients with endometriosis. Pak J Med Sci. 2016; 32: 1268-72. 